\title{
Muséologies
}

Les cahiers d'études supérieures

\section{Institutions culturelles patrimoniales et nouvelles pratiques numériques}

\section{Jean-Pierre Dalbéra}

Volume 3, numéro 2, printemps 2009

URI : https://id.erudit.org/iderudit/1033560ar

DOI : https://doi.org/10.7202/1033560ar

Aller au sommaire du numéro

Éditeur(s)

Association Québécoise de Promotion des Recherches Étudiantes en Muséologie (AQPREM)

ISSN

1718-5181 (imprimé)

1929-7815 (numérique)

Découvrir la revue

Citer cet article

Dalbéra, J.-P. (2009). Institutions culturelles patrimoniales et nouvelles pratiques numériques. Muséologies, 3(2), 36-47.

https://doi.org/10.7202/1033560ar d'utilisation que vous pouvez consulter en ligne.

https://apropos.erudit.org/fr/usagers/politique-dutilisation/ 


\section{Article deux}

\section{Institutions culturelles patrimoniales}

\section{et nouvelles pratiques numériques}

JEAN-PIERRE DALBÉRA A ÉTÉ CHEF DE LA MISSION DE LA RECHERCHE ET DE LA TECHNOLOGIE DU MINISTĖRE DE LA CULTURE ET DE LA COMMUNICATION, DE 1989 À 2004, APRĖS PLUSIEURS ANNÉES AU CENTRENATIONALDE LA RECHERCHE SCIENTIFIQUE (CNRS), QUULLAVAIT INTÉGRÉ EN 1970 POUR MENER DES RECHERCHES EN OPTIOUE DES SOLIDES ET EN TRAITEMENT DIMAGES. DANS LES ANNÉES 1990, ILAÉTÉ UN DES PIONNIERS DELINTERNET CULTUREL FRANÇAIS, A PILOTÉ LE PREMIER PLAN NATIONAL DE NUMÉRISATION DU PATRIMOINE ET PRODUIT DIMPORTANTES COLLECTIONS DE PUBLICATIONS MULTIMÉDIAS SUR LES GRANDS STTES ARCHÉOLOGIOUES ET LES CÉLÉBRATIONS NATIONALES, PRIMÉES DANS DE NOMBREUX FESTIVALS INTERNATIONAUX. IL A REPRÉSENTÉ LA FRANCE DANS LES INSTANCES CHARGÉES DE LA COORDINATION DES POLITIQUUES DE NUMÉRISATION DES PAYS DE L'UNION EUROPÉENNE ET A PARTICIPÉ À DES PROJETS INTERNATIONAUX MAJEURS POUR LE DÉVELOPPEMENT DE LA SOCIETEE DE LINFORMATION, COMME MINERVA ${ }^{[1]}$, STRABON ${ }^{[2]}$ OU MICHAEL ${ }^{[3]}$. IL A ÉTÉ RESPONSABLE DE 2005 À 2008 DU DÉPARTEMENT INFORMATIQUE ET MULTIMÉDIA DU MUSÉE DES CIVILISATIONS DE LEUROPE ET DE LA MÉDITERRANÉE. DEPUIS DÉCEMBRE 2009, LL EST CHARGÉ DUUNE MISSION D'ÉTUDE PAR LE MINISTRE DE LA CULTURE POUR LA CRÉATION DU FUTUR CENTRE NATIONAL DE CONSERVATION DU PATRIMOINE. IL. EST CHERCHEUR ASSOCIÉ AU LABORATOIRE LEOEN ${ }^{[4]}$ DE LUNIVERSITÉ PARIS VIII ET COLLABORE AVEC LE CENTRE GEORGES POMPIDOU, LÉCOLE DU LOUVRE, LE MUSEUM NATIONAL DHISTORE NATURELLE ET LE CNRS POUR SUSCITER DE NOUNELLES FORMES DE COMMUNICATION ET DÉCHANGE AVEC LE PUBLIC DANS LE MONOE SCIENTIFIQUE ET CULTUREL[jean-pierredabera@club-internetfr]

[1]

Ministerial Network for Valorising Activities in Digitisation: <http:/ www. minervaeurope.org $/>$.

[2]

Système dinformation multilingue et multmédia pour le patr" mone culturel et le tourisme euro-méditerranéens: <http:// www.strabon.org/portal>.

\section{[3]}

Muttlingual inventory of Cultural Hertage in Europe: <http://www.michael-culture. org/ff/home>.

\section{[4]}

Laboratoire d'évaluation et de développement pour l'édition numérique < <http://wwwleden. $\arg />$. 


\section{La révolution numérique}

Les technologies numériques ont profondément modifié le fonctionnement de nos sociétés, les modes de travail, d'appropriation des connaissances, d'information, de loisirs culturels et d'échanges entre individus et groupes sociaux.

La toile est devenue en près de 15 ans un "méta-médium", ayant intégré tout ce qui l'a précédée. À la fin de $2008,25 \%$ de la population mondiale est connectée à la toile; en 2012, le taux passera à $30 \%$; les analystes estiment qu'à cette époque il y aura 3 milliards de terminaux connectés, dont $50 \%$ seront des mobiles.

Ces dernières années, en plus des usages traditionnels, la principale évolution sur la toile a été portée par le développement des applications de type Web 2.o. De nouvelles formes de créativité et d'expression personnelle ont émergé, stimulant une culture de l'échange et de la participation. De grands réseaux sociaux comme Facebook, Myspace, Flickr, Youtube, Dailymotion, Second Life... se sont imposés et drainent, dans le monde, des centaines de millions d'utilisateurs, souvent regroupés par affinités ${ }^{[5]}$.

Les transformations qui se sont produites depuis cinq ans obligent les institutions culturelles à redéfinir les relations qu'elles entretiennent avec leur public. Ces institutions se doivent d'être présentes sur Internet et d'y proposer des offres originales. Mais la partie n'est pas gagnée pour autant car leurs offres multimédias sont aujourd'hui en concurrence frontale avec celles de tous les autres secteurs culturels et notamment des médias. Dans un tel contexte, le monde culturel patrimonial doit faire un effort de positionnement stratégique, mettant en valeur ses atouts spécifiques et sa légitimité scientifique, tout en restant attractif et ouvert sur la société. 


\section{La prise de conscience des institutions culturelles françaises}

Expositions virtuelles, catalogues en ligne personnalisables, accès aux documents numérisés, visioconférences, forums de discussion, blogs, sites communautaires ou d'échanges de photographies ou de vidéos, interfaces ${ }_{3} \mathrm{D}$ ont fait évoluer très rapidement les modes d'accès et de partage de l'information. Ainsi, les sites Web des médias, qui permettent grâce au téléchargement de fichiers (en podcasting) d'écouter ou de visionner nombre de leurs émissions sur des dispositifs mobiles, en temps différé et en n'importe quel lieu, sont utilisés par des millions d'internautes.

Les productions visuelles, sonores et audiovisuelles des institutions culturelles ne pouvaient échapper à une telle évolution. Plusieurs institutions culturelles dans les pays anglo-saxons ont, depuis quelques années, mis à la disposition du public leurs audioguides, leurs conférences ou des extraits de leurs films.

En France, ce mouvement a été amorcé avec le projet du «Grand

Versailles numérique" qui, en 2006, a ouvert avec succès le premier grand site gratuit de téléchargement de fichiers sonores et audiovisuels relatifs au château et au parc de Versailles ${ }^{[6]}$.

Comme d'autres musées nationaux désireux d'innover, le Musée des Civilisations de l'Europe et de la Méditerranée ${ }^{[n]}$ (MUCEM) a fait de même, en mars 2007, à l'occasion de son exposition Trésors du quotidien? Europe et Méditerranée ${ }^{[8]}$ à Marseille. Il a ainsi été possible de confirmer l'intérêt du public pour les commentaires sonores, avec près de 60000 fichiers au format $\mathrm{MP}_{3}$ téléchargés au cours de l'exposition sur différents sites Web, dont iTunes.

\section{Le multimédia au service de la muséographie et de la documentation}

À l'intérieur des espaces muséographiques, les technologies permettent la création d'environnements sonores, cartographiques, visuels et interactifs qui facilitent la compréhension des sujets traités. Elles peuvent faire vivre aux visiteurs des expériences sensorielles, faire appel à leur vécu, à leurs émotions ou à leurs savoirs et contribuer à rendre vivant un parcours d'exposition.

La simulation numérique de phénomènes, de comportements, de pratiques sociales, d'espaces urbains, de bâtiments ou de paysages devient d'ores et déjà possible en deux ou trois dimensions pour mieux faire percevoir des situations, des itinéraires, des processus, des techniques ou des environnements anciens ou futurs. De plus en plus de modèles numériques 
3D deviennent disponibles pour des présentations multimédias ${ }^{[0]}$; ces espaces peuvent même être habités par des avatars qui communiquent entre eux et qui sont pilotés par des visiteurs, par exemple dans le monde virtuel «Second Life» ${ }^{[10]}$.

Des dispositifs électroniques communicants (avec la technologie RFID ${ }^{[11}$ par exemple), informés sur la langue, les domaines d'intérêt ou les besoins spécifiques d'un visiteur, peuvent être intégrés par exemple dans le billet d'entrée. Un guide multimédia mobile peut aussi être personnalisé et accompagner un visiteur tout au long de son parcours dans l'institution. De tels dispositifs mobiles, couplés à des lecteurs répartis dans une exposition, peuvent permettre la personnalisation de la médiation et la multiplication des niveaux de lecture. Ils rendent attractifs les parcours conçus pour les jeunes et facilitent l'évaluation des usages ${ }^{[12]}$.

En matière de qualité d'image, la haute définition (HD) est au centre de l'actualité technologique d'aujourd'hui. Les matériels de tournage, de montage, de diffusion et de lecture, comme les programmes de télévision, font leur apparition dans ce nouveau format.

\section{[9]}

Le musée archéologique de Bavay (Nord de la France) dispose, depuis 2004, d'une salle de spectacles interactive, proposant la restitution virtuelle en $3 \mathrm{D}$ et en relief du forum romain antique (<http://www.culture. gouv.fr/culture/editions/documents/cr112_p20.pd†).

\section{[10]}

<http://secondlife.com/>. [11]

RFID : Radio Frequency Identification.

\section{[12]}

<http://www.erasme.org/>.

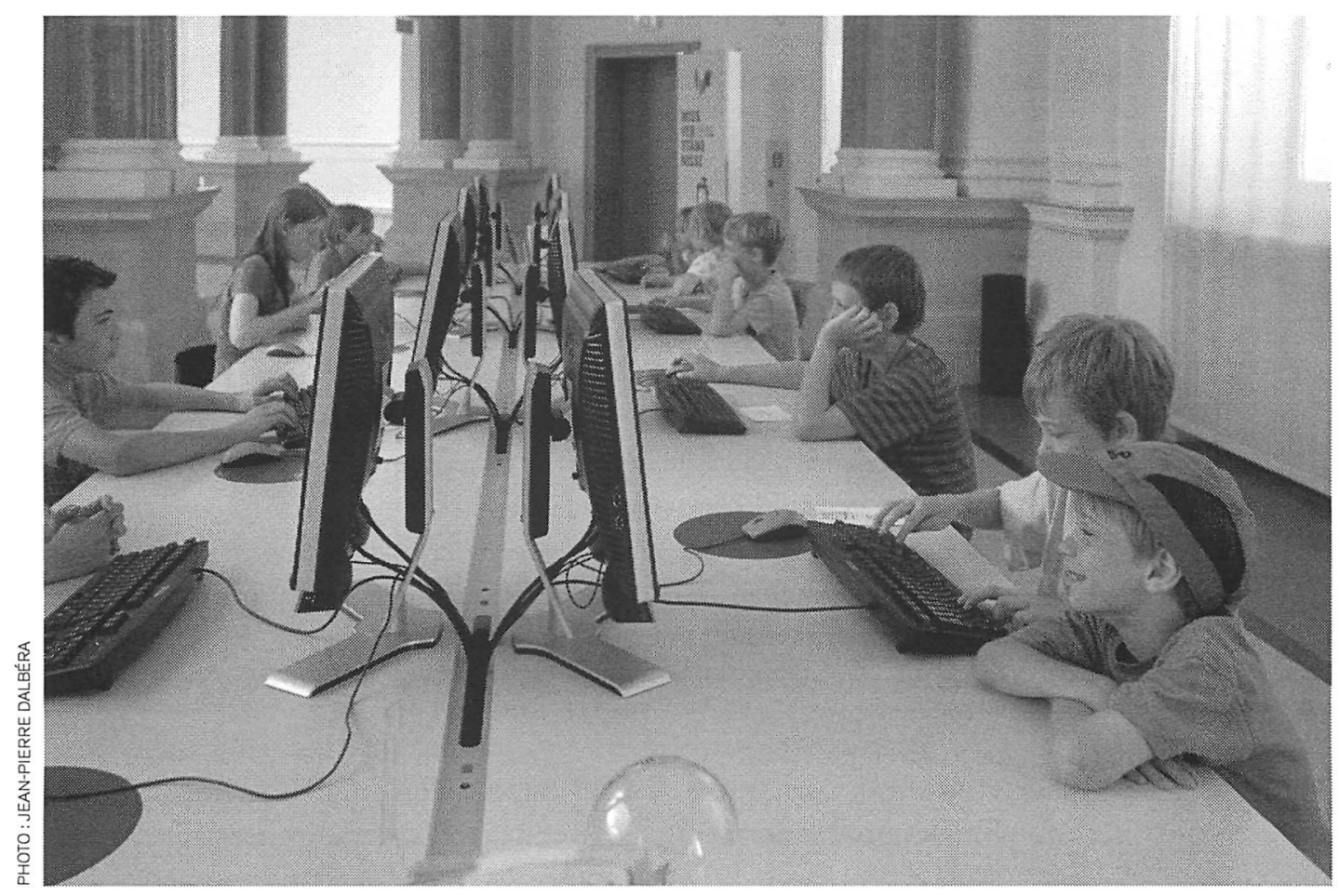

L'espace internet pour les jeunes du musée de la communication de Berlin (Allemagne). 


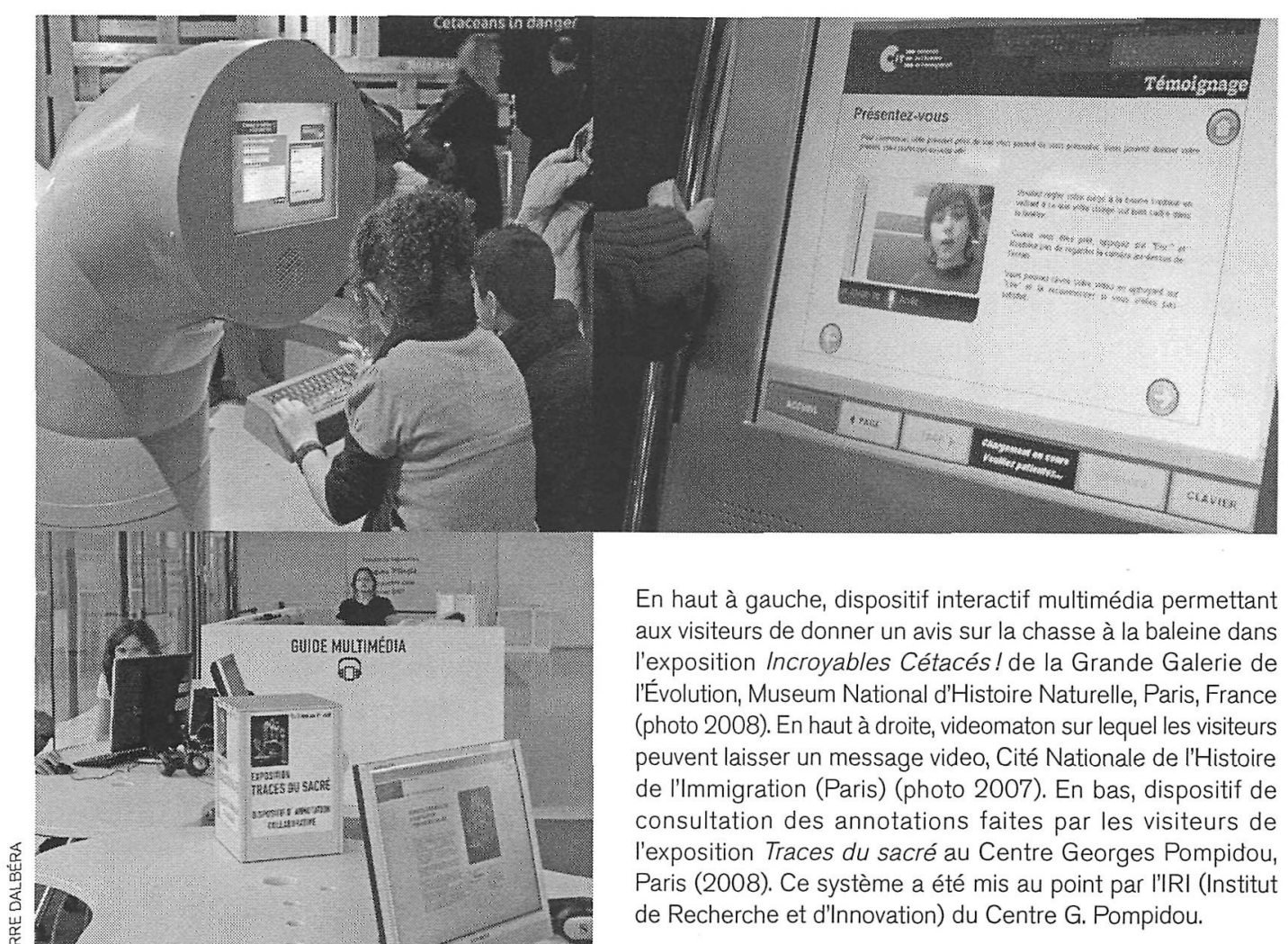

D'ici à 2013, la haute définition constituera le standard de l'offre de télévision et de vidéo à la demande. Parallèlement, l'offre de connexion en fibre optique commence à être proposée aux particuliers. Beaucoup plus

[13]

ADSL: Asymmetric Digital Subscriber Line. rapide et plus stable que l'ADSL ${ }^{[131}$, cette technologie va transformer l'offre de services en associant interactivité, images de haute définition, interfaces $3 \mathrm{D}$ et communication intégrée.

Dans quelques années, elle sera très présente dans les grandes villes et son utilisation fera évoluer les exigences du public en matière de qualité des productions éditoriales et des services multimédias culturels. Dans ce contexte, la visite d'un musée devra rester fortement attractive pour intéresser le public, létonner et le fidéliser. 
Dans le futur, les systèmes informatiques devront être intégrés le plus possible à la scénographie et devenir invisibles. Écrans d'ordinateurs et claviers des bornes d'information actuelles seront amenés à disparaitre; ils seront remplacés par des espaces immersifs, pilotés par des capteurs intégrés aux mobiliers, par des panneaux et des cartels de papier électronique (papiel) destinés à l'affichage et par des systèmes tactiles, ou même sans contact, de sélection des productions éditoriales à la disposition du public.

Dans le centre de ressources d'une institution culturelle, les visiteurs comme les scientifiques devront pouvoir approfondir les sujets de leur choix et consulter tout type de document multimédia. Un tel centre, spécialisé sur le patrimoine, matériel et immatériel, devra proposer des accès aux collections et aux archives scientifiques, sur place et en ligne, mais aussi devenir un lieu de sociabilité, une tête de réseau documentaire, de veille, de collaborations et d'informations échangées. En plus des catalogues et des inventaires, le centre de ressources devra offrir des outils d'annotation des documents multimédias, de traitement des sources en fonction des besoins des utilisateurs et faciliter l'accès à des plateformes d'information et d'échange, $\mathrm{y}$ compris pour les jeunes ${ }^{[14]}$.

\section{Les portails Web}

À la fin des années 1990, les premiers sites Internet des musées ont été conçus comme des plaquettes électroniques au service de la communication, sans exploiter pleinement la valeur ajoutée apportée par l'interactivité et par les réseaux. La majorité des institutions culturelles se sont rapidement rendu compte que ce nouveau média qu'était Internet les obligeait à élargir une vision aussi restrictive et leur ouvrait de nouvelles voies pour communiquer et échanger avec le public.

Aujourd'hui, l'espace Internet d'un musée doit être conçu comme un outil d'information et de documentation, mais aussi d'interpellation du public et de découverte, au même titre qu'une exposition réelle ou une animation culturelle.

Un site Web peut mettre en valeur des collections rarement montrées au public ou apporter de l'information approfondie de tous ordres qui ne trouvent pas leur place dans les expositions réelles. Certaines parties peuvent aussi être destinées aux enfants, à des visiteurs étrangers ou à des publics à besoins spécifiques.
[14]

Le musée de la communication de Berlin privilégie tout particulièrement l'accueil et l'animation multimédia pour les jeunes visiteurs (<http://www. museumsstiftung.de $/$ ). 
Cet objectif implique non seulement que des ressources numériques et des catalogues simplifiés du musée soient diffusés en ligne, mais que des contenus spécifiques et des services soient proposés pour faire du site Web un outil d'incitation à la visite, de fidélisation des visiteurs et d'attractivité pour tous ceux qui habitent loin du musée ou à l'étranger. Ces offres devront de plus en plus être déclinées sous des formats adaptés aux mobiles, comme le proposent déjà certains grands médias.

\section{Le développement de productions éditoriales en ligne}

Dès les débuts de l'Internet culturel, en 1996, une politique éditoriale multimédia, destinée au grand public, a été engagée par la Mission de la recherche et de la technologie (MRT) en collaboration avec la Direction du patrimoine culture/arcnat/fr/>.

[16]

$<$ http://www.culture.gouv.fr/ culture/celebrations/fr/ $>$.

[17] <http://www.ethnologie. culture.fr/>.

[18]

La collection a obtenu le prix Möbius international des multimédias en 2007 à Bucarest et le site "Cornemuses d'Europe et de Méditerranée" a reçu le grand prix AVICOM 2007. du ministère français de la Culture. Elle se poursuit encore aujourd'hui et a toujours pour but principal de faire connaître et de valoriser les recherches sur le patrimoine archéologique soutenues par le ministère.

Ces réalisations éditoriales permettent aux équipes scientifiques d'expérimenter les capacités innovantes des technologies numériques pour expliquer et rendre attractives les connaissances sur le patrimoine. Elles apportent les dimensions multimédias, interactives et ludiques qui manquent aux publications savantes pour susciter l'intérêt du public.

La collection "Grands sites archéologiques " ${ }^{[15]}$ compte aujourd"hui 16 publications, comportant des milliers d'images, de vidéos, de pages de textes, de dessins, d'illustrations, de commentaires sonores. Elle est consultée par des millions de visiteurs et a obtenu plusieurs récompenses internationales: prix Möbius, Webby Award, prix AVICOM.

En 1999, devant le succès de cette formule éditoriale, une nouvelle collection multimédia consacrée aux célébrations nationales ${ }^{[16]}$ a été lancée par la MRT avec la Direction des archives de France. La collection compte aujourd'hui 14 publications.

En 2005, c'est avec le MuCEM en collaboration avec la MRT qu'une troisième collection (appelée "Le portail de l'ethnologie ${ }^{[11]}$ ") a vu le jour pour valoriser les recherches ethnologiques en Europe et en Méditerranée. À ce jour, 8 publications multimédias témoignant de la diversité culturelle ont été produites ${ }^{[18]}$. 
Des politiques éditoriales comparables sont aujourd'hui menées par la plupart des grands musées et des bibliothèques. Complémentaires des catalogues d'expositions édités sur papier, elles sont gratuites et destinées au grand public. Leur présence accroît fortement la richesse de l'offre culturelle sur Internet; ces publications constituent souvent la partie la plus visitée des sites institutionnels.

\section{Les outils du Web 2.0}

L'utilisation du Web s'oriente de plus en plus vers l'interaction avec les utilisateurs et la création de réseaux sociaux. De nombreux sites Web incorporent aujourd'hui des technologies du Web 2.0 pour susciter des échanges autour des sujets traités, que ce soit par écrit ou sous des formes photographiques, sonores ou vidéo.

L'agrégation de contenus est devenue fréquente, grâce à l'utilisation de protocoles standardisés permettant aux utilisateurs qui le souhaitent d'être informés des nouveautés et de faire usage de ces données dans un autre contexte. Grâce à ces technologies, des cercles de "nouveaux amateurs " peuvent se constituer, dont les participants restent en interaction permanente les uns avec les autres et alimentent, à différents niveaux, la réflexion collective ${ }^{[19]}$.

Dans un musée, la participation et l'expression des publics, comme l'instauration de dialogues avec eux, sont des éléments consubstantiels à son existence ${ }^{[20]}$. Ces nouveautés technologiques sont particulièrement adaptées à de tels objectifs. Bien utilisées, elles peuvent permettre au musée de rester en phase avec la société et de répondre à certaines de ses attentes. Peu d'institutions culturelles françaises ont encore vraiment exploré ces voies, mais des expérimentations intéressantes apparaissent ${ }^{\text {t211. }}$.

\section{Vers des plateformes intégrées}

Quatre facettes doivent être prises en compte pour construire une architecture informatique multimédia cohérente dans une institution culturelle: le centre de ressources multimédias, le portail Web, l'intranet de l'institution, les dispositifs muséographiques numériques in situ. Très peu d'institutions procèdent encore de la sorte. Dans une telle perspective, il s'agit d'irriguer les différents espaces réels et virtuels de l'établissement à partir d'un centre de pilotage unique, concentrant l'information, les ressources, les applications métiers, les services et assurant les liaisons avec les réseaux de communication.

\section{[19]}

$<$ http://leden.wordpress.

coms.

[20]

Exemples: le Vidéomaton de la Cité nationale de l'histoire de l'immigration (<http://www. histoire-immigration.fr $>$ ) ou le dispositif interactif de l'exposition Incroyables Cétacés! de la Grande Galerie de l'Évolution (<http://www2.mnhn.fr/ cetaces $/>$ ).

[21]

Exemples: le Muséum de Toulouse est particulièrement présent sur les réseaux sociaux (<http://www.museum. toulouse.fr/ $>$ ), I'Institut de recherche et d'innovation du Centre Georges Pompidou expérimente des outils d'annotation pour les visiteurs à l'occasion d'expositions (<http:// www.iri.centrepompidou.fr $>$ ). 
L'architecture centralisée doit garantir la cohérence d'ensemble d'un tel système d'information, par l'harmonisation des méthodes et des outils de dématérialisation, de gestion des contenus numériques, d'indexation et de documentation, d'archivage, de travail en commun, d'édition, de communication et de services sur la Toile ou sur des supports mobiles. Les modèles de données et de documents numériques doivent être étudiés pour faciliter l'interopérabilité des ressources produites par chaque département de l'établissement afin de parvenir à des gains de productivité et à un fonctionnement interne efficace.

L'enjeu pour la majorité des institutions patrimoniales est, dans un proche avenir, de disposer d'une telle plateforme numérique, développée de préférence sous des logiciels libres, qui puisse maintenir une continuité dans la chaîne de conservation, d'indexation et de production des contenus, intégrant les systèmes d'information spécifiques (pour les collections d'objets, la médiathèque, les archives) jusqu'aux productions éditoriales en ligne, tout en mettant à la disposition des utilisateurs des outils de coopération et d'échanges. C'est à ce prix que les institutions culturelles pourront faire face aux enjeux de la révolution numérique et moderniser en profondeur leurs méthodes de travail et de communication au service des publics. 


\section{Summary}

[Translated by Philippe Lagrange]

The widespread use of digital technologies has deeply modified the modes of operation of patrimonial cultural institutions as regards knowledge, management, and documentation of a historical site or a museum's collection, as well as the information intended for the public and new types of intercommunication that go beyond the passage through the showrooms.

Practically speaking, technologies allow the creation of acoustic, cartographic, visual, and interactive environments, facilitating the comprehension of the different subjects treated. In doing so, they can take the visitors through sensory experiences, appealing to their real-life situations, their emotions or knowledge, and thus enliven the tour. Mobile devices connected to readers installed in the showrooms enable to personalize the multimedia experience, multiplying the reading levels and meeting visitors' specific needs. They make youth-oriented tours attractive and simplify the evaluation of the visitors' habits.

At the institution's resource centre, the guests and scientific personnel must be able to deepen any subject they want. Such a centre must be versatile, focused on cultural heritage, both material and immaterial. It must be a true media library for its collections and resources by giving access to these data both onsite and online. It also should serve as a documentary network leader, a place to socialize, relax, collaborate, and get information on-demand. 
A museum's Webspace ought to be conceived as an informative tool about the activities, researches, and collections; not only should it be attractive to the public, but also a path to discovery, as much as any actual exposition or cultural animation would be. A Website can feature collections rarely shown to the public and provide many levels of knowledge that cannot be added to an actual exposition set. Some parts of the site may be dedicated to children, foreign visitors, or designed for people with specific needs.

All of these technological developments must be taken into consideration in the design and preparation of a new museographic project or the planning of patrimonial sites that will be opened to the public.

In a heritage cultural institution, the ideal digital platform shall comprise four interlinked points that integrate: the multimedia resource centre, the institution's intranet, the Web portal, and the in situ digital device's drivers. 